INPLASY

PROTOCOL

To cite: Sun et al. Effectiveness of Kinesio taping on peripheral facial paralysis: a protocol for systematic review and meta analysis. Inplasy protocol 2020100008. doi:

10.37766/inplasy2020.10.0008

Received: 04 October 2020

Published: 04 October 2020

Corresponding author:

Zai-hui Sun

408128933@qq.com

Author Affiliation:

Chengdu University of

Traditional Chinese Medicine

Support: YYZX2019015.

Review Stage at time of this submission: Data analysis.

Conflicts of interest:

The authors have no conflicts of interest to disclose.

\section{Effectiveness of Kinesio taping on peripheral facial paralysis: a protocol for systematic review and meta analysis}

Sun, ZH¹; Tian, YP2; Tan, YF3; Tao, D4; Li, WB55; Ding, JL6; Ai, SC7.

Review question / Objective: The aim of this meta-analysis of randomized controlled trials is to evaluate the efficacy of Athletic Tape for Facial Paralysis.

Condition being studied: Bell's palsy is a kind of idiopathic acute peripheral facial palsy, it control the muscles of the facial expressions. Although is expected to have a full recovery rate of $70 \%$, but there are still as much as $30 \%$ of patients with left could be disfiguring facial weakness, the symptoms of involuntary movement or continued crying. The most commonly used treatment option is cortical steroids and antiviral drugs. Kinesio Taping, combined with physical therapy, is a kind of safe and promising adjuvant therapy, used in the acute treatment of bell's palsy. However, need further large-scale randomized controlled study to evaluate these complementary intervention in patients with bell's palsy whether full recovery has significant additive or synergistic effect.

INPLASY registration number: This protocol was registered with the International Platform of Registered Systematic Review and Meta-Analysis Protocols (INPLASY) on 04 October 2020 and was last updated on 04 October 2020 (registration number INPLASY2020100008).

\section{INTRODUCTION}

Review question / Objective: The aim of this meta-analysis of randomized controlled trials is to evaluate the efficacy of Athletic Tape for Facial Paralysis.
Condition being studied: Bell's palsy is a kind of idiopathic acute peripheral facial palsy, it control the muscles of the facial expressions. Although is expected to have a full recovery rate of $70 \%$, but there are 
still as much as $30 \%$ of patients with left could be disfiguring facial weakness, the symptoms of involuntary movement or continued crying. The most commonly used treatment option is cortical steroids and antiviral drugs. Kinesio Taping, combined with physical therapy, is a kind of safe and promising adjuvant therapy, used in the acute treatment of bell's palsy. However, need further large-scale randomized controlled study to evaluate these complementary intervention in patients with bell's palsy whether full recovery has significant additive or synergistic effect.

\section{METHODS}

Participant or population: The inclusion criteria is accord with the standard of clinical diagnosis of the Facial Paralysis of adults.

Intervention: Athletic Tape was the main intervention.

Comparator: Other treatments besides athletic tape was the main intervention (e.g. acupuncture, drugs and Physical therapy).

Study designs to be included: Randomized controlled trials (RCTs) and controlled clinical trials (CCTs) will be included.

Eligibility criteria: Interventions will include any type of Kinesio taping for improvement of symptoms of peripheral facial paralysis.

Information sources: We will search the following electronic databases for relevant trials from inception to present: China National Knowledge Infrastructure(CNKI), Wanfang Date, SinoMed, Technology Periodical Dat abase (VIP), PubMed,Embase, Web of Science and The Cochrane Library.

Main outcome(s): The primary outcome will be the total effective rate.

Additional outcome(s): Secondary outcomes will include House-Brackmann scale, Portmann score, facial nerve conduction velocity (NCV), Facial Disability
Index (FDI), Facial Disability Index include Facial Function score (FDIp) and social Function score (FDIs).

Data management: Two authors will independently extract data. Any disagreement will be resolved by discussion until consensus is reached or by consulting a third author.

Quality assessment / Risk of bias analysis: The risk of bias of included studies was assessed by using an assessment tool, the Cochrane Handbook for Systematic Reviews of Interventions version 5.1.0 (updated in 2011 by the Cochrane Organization) by 2 reviewers subjectively. The assessment tool covers 7 domains: random sequence generation (selection bias), allocation concealment (selection bias), blinding of participants and personnel (performance bias), blinding of outcome assessment (detection bias), incomplete outcome data (attrition bias), selective reporting (reporting bias), and other biases. Bias were assessed as low risk, high risk, or unclear risk. Discrepancies were resolved through discussion and settled by a third reviewer.

Strategy of data synthesis: The statistical analyses software, Revman 5.3, was used to conduct this meta-analysis.Differences in mean, standard deviation, sample size, with $95 \%$ confidence interval were measured to compare the Kinesio taping group with control group to investigate the changes in Therapeutic efficiency, Portmann scores, Facial Disability Index (FDI). Statistical heterogeneity between studies was tested using $I^{2}$ statstics, which presented the percentage of the total variability among the studies that was caused by heterogeneity rather than chance. The 2 test was used to assess whether differences in results were compatible with chance alone, a Pvalue $\leq 0.1$ was considered to indicate significant heterogeneity. If $P>0.1$ and $\left.\right|^{2}<50 \%$, statistical heterogeneity was perceived to be acceptable and a fixed effects model was used to conduct the meta-analysis; otherwise, if $P<0.1$ and 
$I^{2} \geq 50 \%$, statistical heterogeneity was perceived to be relatively high and a random effects model was used to perform the meta-analysis.

Subgroup analysis: Subgroup analysis and sensitivity analysis were further conducted to explore the source of such heterogeneity. If $P \geq 0.1$ studies with the same outcome were included, a funnel plot was used to assess publication bias of the included studies.

Sensibility analysis: The results of one or more outlier studies will conflict with other studies and may become a source of heterogeneity. In order to ensure the quality of Meta-analysis we will perform a sensitivity analysis to exclude outliers.

Language: Without any language or publication status restrictions.

Country(ies) involved: China.

Keywords: Kinesio taping, peripheral facial paralysis, meta-analysis, systematic review, protocol.

Dissemination plans: The results of this systematic review will be published in a peer-reviewed journal or presented at conferences.

Contributions of each author:

Author 1 - Zai-hui Sun - Author 1 drafted the manuscript.

Author 2 - Yan-ping Tian - The author provided statistical expertise.

Author 3 - Yan-fu Tan - The author contributed to the development of the selection criteria, and the risk of bias assessment strategy.

Author 4 - Dan Tao - The author contributed to the development of the selection criteria, and the risk of bias assessment strategy.

Author 5 - Wen-bo Li - The author ACTS as a supervisor.

Author 6 - Ji-lin Ding - The author contributed to the development of the selection criteria, and the risk of bias assessment strategy.
Author 7 - Shuang-chun Ai - The author read, provided feedback and approved the final manuscript. 